Discussion Paper No. 869

\title{
SHOULD FIRMS EMPLOY PERSONALIZED PRICING?
}

Toshihiro Matsumura

Noriaki Matsushima

March 2013

The Institute of Social and Economic Research Osaka University

6-1 Mihogaoka, Ibaraki, Osaka 567-0047, Japan 


\title{
Should firms employ personalized pricing?*
}

\author{
Toshihiro Matsumura ${ }^{\dagger}$ \\ Institute of Social Science, the University of Tokyo \\ Noriaki Matsushima ${ }^{\ddagger}$ \\ Institute of Social and Economic Research, Osaka University
}

\begin{abstract}
The recent developments in information technology (IT) have enabled firms to employ personalized pricing. Should all firms employ personalized pricing even though the adaptation costs of such pricing strategies are not high? This paper theoretically demonstrates a situation in which all firms do not always employ personalized pricing even though the fixed costs to do so is zero. The model is based on those of Thisse and Vives (1988) and Shaffer and Zhang (2002). Our model incorporates the fact that firms engage in marginal costreducing activities after they decide whether to employ personalized pricing. As employing personalized pricing induces the rival firm to engage more in reducing its costs, to mitigate the cost-reducing activities of firms, the less-efficient firm should not employ personalized pricing. Our main result indicates that such firms should take into account their relative competitive positions and technological environments. When firms are small, they would need to reconsider whether to employ personalized pricing.
\end{abstract}

JEL codes: M31, L13, D43

Keywords: personalized pricing, heterogeneity, cost reductions, game theory

* We would like to thank seminar participants at Nagoya University and Shinshu University for their helpful comments. The authors gratefully acknowledge financial support from Grant-in-Aid from the Japanese Ministry of Education, Science and Culture. Needless to say, we are responsible for any remaining errors.

${ }^{\dagger}$ Toshihiro Matsumura, Institute of Social Science, the University of Tokyo, Hongo 7-3-1, Bunkyo, Tokyo 113-0033, Japan.

${ }_{\ddagger}^{\ddagger}$ Corresponding author: Noriaki Matsushima, Institute of Social and Economic Research, Osaka University, Mihogaoka 6-1, Ibaraki, Osaka 567-0047, Japan. Phone: +81-6-6879-8571. E-mail: nmatsush@iser.osaka-u.ac.jp 


\section{Introduction}

The recent developments in information technology (IT) have enabled firms to employ personalized pricing (Arora et al., 2008 and references therein). For instance, by using profiles developed by information collected through loyalty cards, Tesco learns a variety of facts about what and when its customers buy. Tesco's Clubcard operation uses these data to send personalized coupons and other offers to every Clubcard household in each quarter, a huge operation that accounts for more than $6 \%$ of the U.K.'s annual postal volume (Raju and Zhang, 2010, p.131-2). ${ }^{1}$ However, some companies have not employed such personalized pricing strategies even though they potentially could. A simple explanation for this asymmetry concerning personalized pricing is that personalization is expensive and requires data and expensive software to implement (Arora et al., 2008). Although this explanation is plausible, it raises the following question: Should all firms employ personalized pricing even though the adaptation costs of such pricing strategies are not high? This is a reasonable question because the recent rapid development of IT will allow firms to inexpensively employ such a pricing policy in the near future. The purpose of this paper is to provide a different, yet simple rationale behind why some firms do not employ personalized pricing even though personalization is inexpensive.

The model setting is as follows. Two firms compete in a differentiated product market. Each firm determines whether to employ personalized pricing. If a firm employs personalized pricing, it will be able to distinguish among customers and offer them personalized prices; otherwise, it offers a standard non-discriminatory price to all customers. The model setting is based on those of Thisse and Vives (1988) and

\footnotetext{
${ }^{1}$ Recently, grocers like Safeway and Kroger also began offering different methods to determine individualized prices (The New York Times (August 10,2012)). Hoping to improve razor-thin profit margins, they are creating specific offers and prices, based on shoppers' behaviors, which could encourage them to spend more.
} 
Shaffer and Zhang (2002). Our model also incorporates the fact that firms engage in marginal cost-reducing activities. More concretely, we set a model in which the firms engage in these activities after they determine their pricing policies. In the retailing context, for instance, retailers need to construct electric devices to distribute appropriate promotional coupons through frequent-shopper programs based on the analyses of household purchase records. Furthermore, to use such devices, these retailers also need new cash registers, computers for gathering the records of customer purchases, applications for analyzing the records, data analysts, customer service offices for their frequent shopper programs, and so on. For instance, issuing promotional coupons for each customer generates an additional per-customer cost. If the retailer creates a device to incorporate promotional coupons into every customer's card, it would save this additional per-customer cost despite possible additional running costs. Introducing such a new device is an example of a cost-reducing activity by retailers. Introducing personalized pricing is time consuming, requires much time to make the pricing policy work well, and thus is a long-term decision, even though the cost of developing personalized pricing is low. We therefore believe that firm should make their pricing policy decisions before they engage in cost-reducing activities.

Even if a firm does not employ personalized pricing, it still engages in cost-reducing activity. $^{2}$ One would think that a firm can keep its R\&D costs down by not employing personalized pricing. Although we first discuss the case in which the effort costs under

\footnotetext{
${ }^{2}$ This case is similar to oligopoly models with cost reductions where firms engage in cost-reducing activities before they set their prices or quantities, which have been intensively discussed in the literature of industrial organization (e.g., Brander and Spencer, 1983). Recently, researchers have investigated the relationship between firm asymmetry and R\&D activities as discussed in this paper (Cohen and Klepper, 1996; Matsumura and Matsushima, 2010, Ishida et al., 2011). We believe that our paper can make a new contribution in this context. Although most studies in this literature focus on how R\&D affects equilibrium pricing levels, our paper sheds light on how each firm's decision on pricing policy (whether to employ personalized pricing) affects the strategic R\&D behavior of firms, and how the degree of firm asymmetry matters.
} 
the two pricing policies are the same in Section 4.1, we also discuss how the main result in Section 4.1 changes if the costs of cost-reducing activities under the two pricing policies differ in Section 4.2.

We show that when the ex ante cost difference between the firms is large, the lessefficient firm does not employ a personalized pricing policy even though the fixed cost to employ the policy is zero, while the more efficient firm employs the personalized pricing policy. The key point is strategic interaction between the less-efficient firm's pricing policy and its rival's cost-reducing activity. In fact, this asymmetric result does not hold when the firms do not engage in cost-reducing activities. ${ }^{3}$

We explain the intuition behind why personalized pricing is not always adopted. Let us first consider the case in which no firm employs a personalized pricing policy, that is, two firms adopt a standard uniform pricing policy. As in the standard price competition with differentiated products, a firm's cost reduction leads to a reduction in its price. Through the strategic interaction between the firms (due to the strategic complementarity of prices), the price reduction induces a lower price in the rival firm, which reduces the profit of the cost-reducing firm. Thus, under a standard uniform pricing policy, each firm has a smaller incentive to carry out cost-reducing activities than if the cost reduction had no strategic effect on the rival's pricing.

Let us next consider the case in which both firms employ a personalized pricing policy. The competition in each customer market is similar to that of the standard Bertrand competition. Only the firm with a cost advantage to the rival supplies for each market, and then the price is equal to the rival's marginal cost for the market it supplies. Although a cost reduction by a firm reduces its rival's prices in the rival's target markets, the cost reduction does not affect the cost-reducing firm's prices in its

\footnotetext{
${ }^{3}$ In the context of marketing, Moorthy (2005) focuses on retail price changes motivated by cost changes.
} 
target markets. This suggests that a unilateral cost reduction will be more profitable under a personalized pricing policy than under a uniform pricing policy. Note that the larger the firm's market share, the greater incentive it has for cost-reducing activity under a personalized pricing policy.

Let us finally suppose that one of the firms (firm 2) unilaterally deviates from a personalized pricing policy and employs uniform pricing. Then, firm 2's uniform price is now dependent on the cost of its rival (firm 1), and thus, firm 1's prices in the target markets are also dependent on its own cost. A reduction in firm 1's cost reduces firm 1's prices through the reaction of firm 2, in contrast to the situation where both firms employ personalized pricing. Thus, firm 1 has a weaker incentive for cost reduction when firm 2 employs a uniform pricing policy, which mitigates competition between them and increases the profit of firm 2. As we explained, a firm with a larger market share (firm 1) has a stronger incentive for cost-reducing activity. Moreover, the negative effect of firm 1's cost reduction through the reaction by firm 2 increases with the market share of firm 1 because a price decrease caused by price competition, ceteris paribus, harms the larger firm (firm 1) more. These suggest that a unilateral deviation from a personalized pricing policy by firm 2 reduces firm 1's cost-reducing activity more when firm 1's market share is larger. If the market share of firm 1 is sufficiently large, then at some point, firm 2 actually prefers adopting a uniform pricing policy in order to dampen the incentive of firm 1 with a larger market share to engage in cost-reducing activities. This is why the less-efficient firm is motivated to adopt uniform pricing rather than personalized pricing.

We review related research and discuss the relation between these papers and ours. Recent studies emphasize that the promise of personalization enables firms to estimate their customers' valuations and thus implement finer price discrimination (Shaffer and Zhang, 1995, 2000, 2002; Bester and Petrakis, 1996; Villas-Boas, 1999; Fudenberg and 
Tirole, 2000; Chen and Iyer, 2002; Desai and Purohit, 2004; Bhaskar and To, 2004; Liu and Serfes, 2004; Taylor, 2004; Liu and Zhang, 2006; Dogan et al., 2010; Shulman and Geng, 2012). ${ }^{4}$ Among the existing literature, some papers have already investigated why asymmetry concerning the employment of personalized pricing occurs and have presented interesting and plausible answers (Shaffer and Zhang, 2002; Choudhary et al., 2005; Ghose and Huang, 2009). Shaffer and Zhang (2002) introduce firm heterogeneity into a spatial competition model with price discrimination and show that personalized pricing does not always lead to a prisoner's dilemma situation; that is, a firm in an advantageous position can benefit from personalized pricing. They also show that only the advantageous firm employs personalized pricing when the per customer cost of offering a personalized price is moderate. However, they do not explicitly discuss whether each firm commits to employing personalized pricing. Moreover, our model incorporates the effort choices of firms to improve their production environments. Choudhary et al. (2005) examine a vertically differentiated duopoly and show that the higher-quality firm can be worse off with personalized pricing. The driving force of their main result is as follows. Personalized pricing employed by a firm motivates its rival to improve its product quality. The effect of the quality improvement depends on who employs it. When the rival is the high-quality firm, that is, when the low-quality firm employs personalized pricing, the quality improvement by the rival (the high quality firm) mitigates competition because the quality differentiation between the two firms increases. The converse also holds true. When the rival is the low-quality firm, that is, when the high-quality firm employs personalized pricing, the quality improvement by the rival accelerates competition because the quality differentiation between the two firms decreases. ${ }^{5}$ In our model, quality improvements (which

\footnotetext{
${ }^{4}$ Arora et al. (2008) and Zhang (2009) provide excellent surveys of the discussion of personalized pricing.

${ }^{5}$ Moreover, when the high-quality firm employs personalized pricing to escape the intense compe-
} 
have qualitatively similar effects with cost reductions) by both the high-quality and the low-quality firms accelerate competition, which yields the contrasting result in which the higher-quality firm is always better off with personalized pricing. This property is quite different from that of Choudhary et al. (2005). Ghose and Huang (2009) also incorporate the efforts of firms to improve product qualities into duopoly competition with personalized pricing. ${ }^{6}$ Shaffer and Zhang (2002) show that a prisoner's dilemma situation does not arise even when the firms are ex ante symmetric, but they do not explicitly discuss whether each firm commits to employing personalized pricing.

The paper proceeds as follows. The next section outlines the basic environment. Section 3 shows the benchmark result. Section 4 elaborates on the basic environment and displays the main result. Finally, Section 5 concludes the paper.

\section{Basic environment}

Before we discuss the main concern of our paper, we explain the model without costreducing activities. In Section 4, we explicitly discuss our main concern.

Consider a linear city along the unit interval [0,1], where firm 1 is located at 0 and firm 2 is located at 1 . Consumers are uniformly distributed along the interval, and the density of the consumer distribution is 1. Each consumer buys exactly one unit of the good, which can be produced by either firm 1 or firm 2. A consumer locating at $x \in[0,1]$ incurs a transportation cost of $t x(t(1-x))$ when purchasing a product from firm 1 (firm 2), where $t$ is a positive constant. Each consumer derives a surplus from consumption (gross of price and transportation costs) equal to $v$. We

tition through the additional investment by the low-quality firm, an additional quality improvement is needed for the high-quality firm. The additional investment cost is high because the marginal investment cost of the high-quality firm is high. This additional cost is not compensated by the gain from personalized pricing.

6 Product customization is also a means to discriminate between customers. Alptekinoğlu and Corbett (2008) discuss product customization in duopoly competition. 
assume that $v$ is so large that every consumer consumes one unit of the product. We think that the model reflects the spatial nature of competition, which is applicable to many markets including competition among retailers in cities, manufacturers with differentiated products, and so on.

The firms produce homogeneous goods with the constant marginal cost $c_{i}>0$ $(i=1,2)$. We assume that $t>\left|c_{2}-c_{1}\right|$ to ensure that the quantity supplied by the firms is always positive. Let $p_{i}(x)$ denote the price of firm $i(i=1,2)$, where $x$ is a point on the interval $[0,1]$. That is, the prices set by the firms depend on point $x \in[0,1]$.

Given that firms 1 and 2 set $p_{1}(x)$ and $p_{2}(x)$, respectively, consumer $x$ buys a good from firm 1 if and only if $p_{1}(x)+t x \leq p_{2}(x)+t(1-x)$; note that if $p_{1}(x)+t x=$ $p_{2}(x)+t(1-x)$, the consumer randomly chooses one of the goods.

Each firm has two options to set its price $p_{i}(x)$. The first is uniform pricing ("UP"). When firm 1 (firm 2) employs UP, its price strategy is given by $p_{1}(x)=p_{1}\left(p_{2}(x)=p_{2}\right)$, where $p_{i}$ is a constant value set by firm $i(i=1,2)$. We interpret $p_{i}$ as firm $i$ 's regular price which is common for all consumers. The second is a targeted pricing ("TP"). When firm $i$ employs TP, it modifies its price at each point $x$ even after setting its regular price $p_{i}$. We interpret the modification of the price at each point $x$ as promotion strategies of firm $i$. Note that we assume that information about the location of each consumer is available to the firms. Firms can obtain such information from a number of sources, such as through repeated past transactions with the customers (Liu and Serfes, 2004). ${ }^{7}$

The game runs as follows. First, each firm simultaneously determines its pricing policy, UP or TP. Second, after observing the rival's pricing policy, each firm simultaneously sets its regular price. If firm $i$ has chosen UP, it is its final price. Third, if firm $i$

\footnotetext{
${ }^{7}$ Liu and Serfes (2004) and Shaffer and Zhang (2000, 2002) provide more detailed discussions on this issue.
} 
has employed TP, it modifies its price at each point $x$. If the two firms have employed TP, they simultaneously modify their prices at each point $x$. The solution concept is the subgame perfect Nash equilibrium. We therefore solve the game by backward induction.

The timing structure in our model follows that in the related papers (Banks and Moorthy, 1999; Liu and Serfes, 2004; Rao, 1991; Shaffer and Zhang, 1995, 2002; Thisse and Vives, 1988; Zhang, 2009). These papers assume that firms choose their promotional strategies after they have chosen their regular prices. This assumption reflects the common view that a firm's choice of regular price can be adjusted slower than its choice of promotional offers such as targeted coupons. In addition, if both decisions are made simultaneously, no pure strategy equilibrium exists in the subgames where only one firm employs TP.

\section{Benchmark analysis without cost-reducing activ- ities}

There are four cases depending on the pricing strategies set by the firms. We discuss each of the four cases.

UP-UP: When both firms employ UP, the indifferent consumer, $\hat{x}_{n n}$, is given as

$$
p_{1}+t \hat{x}_{n n}=p_{2}+t\left(1-\hat{x}_{n n}\right) \rightarrow \hat{x}_{n n}=\frac{t+p_{2}-p_{1}}{2 t}
$$

The profits are given by

$$
\pi_{1}=\left(p_{1}-c_{1}\right) \hat{x}_{n n}, \quad \pi_{2}=\left(p_{2}-c_{2}\right)\left(1-\hat{x}_{n n}\right) .
$$

The first-order conditions lead to

$$
p_{i}=\frac{3 t+2 c_{i}+c_{j}}{3}, \quad \hat{x}_{n n}=\frac{3 t-c_{1}+c_{2}}{6 t}, \quad \pi_{i}=\frac{\left(3 t-c_{i}+c_{j}\right)^{2}}{18 t} .
$$


UP-TP: Consider the case in which firm 1 employs UP and firm 2 employs TP. We can apply the discussion of this case to that in which firm 2 employs UP and firm 1 employs TP. When only firm 1 employs UP, the timing is as follows. First, firm 1 sets its price. Second, observing this price, firm 2 sets its prices.

Given that firm 1 sets its price $p_{1}$, firm 2 takes the demand of consumer $x$ if and only if

$$
p_{1}+t x>p_{2}(x)+t(1-x) \quad \rightarrow \quad p_{2}(x)<p_{1}+t(2 x-1) .
$$

If the right-hand side in the latter inequality is larger than the marginal cost of firm $2, c_{2}$, it sets $p_{2}(x)$ at $p_{1}+t(2 x-1)-\varepsilon$ to take the demand of consumer $x$, where $\varepsilon$ is sufficiently small; we omit this value. Otherwise, it sets $p_{2}(x)$ at $c_{2}$. The optimal price of firm 2 at point $x$ is given by

$$
p_{2}(x)= \begin{cases}p_{1}+t(2 x-1) & \text { if } c_{2}<p_{1}+t(2 x-1) \\ c_{2} & \text { otherwise }\end{cases}
$$

The indifferent consumer $\hat{x}_{n d}$ is given by

$$
c_{2}=p_{1}+t(2 x-1) \rightarrow \hat{x}_{n d}=\frac{t+c_{2}-p_{1}}{2 t} .
$$

Anticipating the prices of firm 2, firm 1 sets its price, $p_{1}$. The profit of firm 1 is given by

$$
\pi_{1}=\left(p_{1}-c_{1}\right) \hat{x}_{n d}=\frac{\left(p_{1}-c_{1}\right)\left(t+c_{2}-p_{1}\right)}{2 t} .
$$

The first-order condition leads to

$$
p_{1}=\frac{t+c_{1}+c_{2}}{2}, \quad \hat{x}_{n d}=\frac{t+c_{2}-c_{1}}{4 t}, \quad \pi_{1}=\frac{\left(t+c_{2}-c_{1}\right)^{2}}{8 t}
$$

The profit of firm 2 is given by

$$
\pi_{2}=\int_{\hat{x}_{n d}}^{1}\left(p_{1}+t(2 m-1)-c_{2}\right) d m=\frac{\left(3 t+c_{1}-c_{2}\right)^{2}}{16 t} .
$$


Conversely, under the case in which firm 2 employs UP and firm 1 employs TP, the profits of the firms are given by

$$
\pi_{1}=\frac{\left(3 t+c_{2}-c_{1}\right)^{2}}{16 t}, \quad \pi_{2}=\frac{\left(t+c_{1}-c_{2}\right)^{2}}{8 t} .
$$

TP-TP: When both firms employ TP, the lowest price of firm $i$ for each consumer is $c_{i}$. Firm 1 takes the demand of consumer $x$ if and only if

$$
p_{1}(x)+t x<c_{2}+t(1-x) \rightarrow p_{1}(x)<c_{2}+t(1-2 x) .
$$

If the right-hand side in the latter inequality is larger than the marginal cost of firm $1, c_{1}$, it sets $p_{1}(x)$ at $c_{2}+t(1-2 x)-\varepsilon$ to take the demand of consumer $x$, where $\varepsilon$ is sufficiently small; we omit this value. Otherwise, it sets $p_{1}(x)$ at $c_{1}$. The optimal price of firm 1 at point $x$ is given by

$$
p_{1}(x)= \begin{cases}c_{2}+t(1-2 x) & \text { if } c_{1}<c_{2}+t(1-2 x) \\ c_{1} & \text { otherwise }\end{cases}
$$

A similar argument is applied to the prices of firm 2 .

$$
p_{2}(x)= \begin{cases}c_{1}+t(2 x-1) & \text { if } c_{2}<c_{1}+t(2 x-1) \\ c_{2} & \text { otherwise }\end{cases}
$$

The indifferent consumer $\hat{x}_{d d}$ is given by

$$
c_{1}+t x=c_{2}+t(1-x) \rightarrow \hat{x}_{d d}=\frac{t+c_{2}-c_{1}}{2 t}
$$

The profits of the firms are given by

$$
\begin{aligned}
& \pi_{1}=\int_{0}^{\hat{x}_{d d}}\left(c_{2}+t(1-2 m)-c_{1}\right) d m=\frac{\left(t+c_{2}-c_{1}\right)^{2}}{4 t}, \\
& \pi_{2}=\int_{\hat{x}_{d d}}^{1}\left(c_{1}+t(2 m-1)-c_{2}\right) d m=\frac{\left(t+c_{1}-c_{2}\right)^{2}}{4 t} .
\end{aligned}
$$


Table 1: Payoff matrix when the marginal costs are exogenous

\begin{tabular}{c|c|cc}
$1 / 2$ & $\mathrm{UP}$ & \multicolumn{2}{|c}{$\mathrm{TP}$} \\
\hline $\mathrm{UP}$ & $\frac{\left(3 t+c_{1}-c_{2}\right)^{2}}{18 t}$ & $\frac{\left(3 t+c_{1}-c_{2}\right)^{2}}{16 t}$ \\
$\mathrm{TP}$ & $\frac{\left(3 t+c_{2}-c_{1}\right)^{2}}{18 t}$ & $\frac{\left(t+c_{2}-c_{1}\right)^{2}}{8 t}$ & \\
\hline & $\frac{\left(3 t+c_{1}-c_{1}\right)^{2}}{8 t}$ & $\frac{\left(t+c_{1}-c_{2}\right)^{2}}{4 t}$ & \\
\hline
\end{tabular}

Note: In each cell, the lower left and the upper right items are the payoffs of firms 1 and 2 , respectively.

Payoff matrix: From the above discussion, we obtain the payoff matrix in Table 1.

From the payoff matrix in Table 1, we obtain the following lemma.

Lemma 1. For any $c_{1}$ and $c_{2}$, employing TP dominates employing UP.

This property follows the results discussed in Thisse and Vives (1988) and Zhang (2009). Note that as in Shaffer and Zhang (2002), if $c_{2}-c_{1}$ is large, firm 1's profit in the case in which both firms employ TP is larger than that in which no firm does.

Before we explain the key point of the main result presented in the next section, we review the intuition why both firms employ a personalized pricing policy without costreducing activities, which is shown by Thisse and Vives (1988) and Shaffer and Zhang (2002). Personalized pricing allows a firm to price-discriminate among customers. The firm employing a personalized pricing policy can give special discounts to targeted customers without changing the prices for other customers whose loyalty for the firm is high. On the contrary, under uniform pricing policy, in order to get targeted customers, the firm employing this pricing policy must provide discounts for all customers including those who would buy without discounts. Thus, firms have incentives to employ a 
personalized pricing policy.

Because the effect of price discrimination does not depend on the assumptions that total demand is inelastic and/or all consumers have the same demand, we believe that Lemma 1 holds under broader situations than what we discuss in this paper.

\section{Cost reduction}

The result in the previous section changes when we take into account the cost-reducing activities of firms. Note that we can derive a qualitatively similar result even though the difference between the products is in quality and the firms engage in quality-improving activities. We discuss it after we show the main result.

We consider the following game. First, each firm decides its pricing policy. Second, observing these pricing policies, each firm engages in its cost-reducing efforts. Third, each firm simultaneously sets its regular price. If firm $i$ has chosen UP, it is its final price. Finally, if firm $i$ has employed TP, it modifies its price at each point $x$. If the two firms have employed TP, they simultaneously modify their prices at each point $x$. Under this scenario, the above payoff matrix represents the outcomes after the third stage in the four subgames that follow the first stage.

We have implicitly assumed that the pricing policy decision is time consuming and a long-term one; in other words, it has an investment nature. As mentioned in the Introduction, firms need to spend enough time to construct the IT system for TP. Then, after the construction of the IT system, firms employing the IT system improve the efficiencies of their operations and services that are suitable for the system. Of course, even though a firm does not employ $\mathrm{TP}$, it engages in marginal cost reducing activity after it determines not to employ TP. Furthermore, because the timing structure is also employed in Choudhary et al. (2005), our result in this section is comparable to 
that in Choudhary et al. (2005).

Following the pricing policy employed in the first stage, each firm engages in its efforts to reduce the operation cost. If the firm employs TP, the cost-reducing activities include activities like the stabilization of computer systems for TP and efforts to learn how to use these systems efficiency. If the firm employs UP, the cost-reducing activities are related to the improvement of its daily activities. Under this interpretation, one may think that employing TP requires much effort to make the operations of the firm work well. To clarify the logic behind our main result, we first assume that the effort costs of firm $i$ to reduce $c_{i}$ are common in the two pricing policies. We also discuss a case in which the effort costs under the two pricing policies differ after we investigate the symmetric case.

\subsection{Symmetric effort costs}

We first consider the case in which the effort costs to decrease the marginal cost do not depend on the decision of pricing policy.

We now investigate how $c_{1}$ and $c_{2}$ are determined by the firms. Assume that the ex ante marginal costs of firms are $c_{1}=c-e_{1}$ and $c_{2}=c+d-e_{2}$, where $c$ and $d$ are positive constants and $e_{i}$ is firm $i$ 's effort to reduce its marginal cost $(i=1,2)$. From above, we have that firm 1 is more efficient than firm 2. $I\left(e_{i}\right)$ is the effort cost of firm $i$. We assume that $I\left(e_{i}\right)=\gamma e_{i}^{2}$, where $\gamma$ is a positive constant. For simplicity, we assume that the effort costs under the two pricing policies are the same. This assumption clarifies the effects of pricing policy on the efforts and prices in the second and third stages. To guarantee that the second-order conditions for cost-reducing activities are satisfied, we assume that $t \gamma \geq 1$.

We discuss the four cases in the main text: (1) both firms employ TP; (2) only firm 1 employs TP; (3) only firm 2 employs TP; and (4) no firm employs TP. 
Both firms employ TP: The profits in the second stage are given as

$$
\pi_{1}=\frac{\left(t+d-e_{2}+e_{1}\right)^{2}}{4 t}-\gamma e_{1}^{2}, \quad \pi_{2}=\frac{\left(t-d-e_{1}+e_{2}\right)^{2}}{4 t}-\gamma e_{2}^{2} .
$$

The first-order conditions yield the following reaction functions of the firms:

$$
e_{1}\left(e_{2}\right)=\frac{t+d-e_{2}}{4 t \gamma-1}, \quad e_{2}\left(e_{1}\right)=\frac{t-d-e_{1}}{4 t \gamma-1} .
$$

Solving these equations, we have

$$
\begin{aligned}
& e_{1}=\frac{2 t \gamma+2 d \gamma-1}{4 \gamma(2 t \gamma-1)}, \quad e_{2}=\frac{2 t \gamma-2 d \gamma-1}{4 \gamma(2 t \gamma-1)} \\
& \pi_{1}=\frac{(4 t \gamma-1)(2 t \gamma+2 d \gamma-1)^{2}}{16 \gamma(2 t \gamma-1)^{2}}, \quad \pi_{2}=\frac{(4 t \gamma-1)(2 t \gamma-2 d \gamma-1)^{2}}{16 \gamma(2 t \gamma-1)^{2}}
\end{aligned}
$$

Only firm 1 employs TP: The profits in the second stage are given as

$$
\pi_{1}=\frac{\left(3 t+d-e_{2}+e_{1}\right)^{2}}{16 t}-\gamma e_{1}^{2}, \quad \pi_{2}=\frac{\left(t-d-e_{1}+e_{2}\right)^{2}}{8 t}-\gamma e_{2}^{2} .
$$

The first-order conditions yield the following reaction functions of the firms:

$$
e_{1}\left(e_{2}\right)=\frac{3 t+d-e_{2}}{16 t \gamma-1}, \quad e_{2}\left(e_{1}\right)=\frac{t-d-e_{1}}{8 t \gamma-1}
$$

Solving these equations, we have

$$
\begin{aligned}
& e_{1}=\frac{6 t \gamma+2 d \gamma-1}{2 \gamma(16 t \gamma-3)}, \quad e_{2}=\frac{4 t \gamma-4 d \gamma-1}{2 \gamma(16 t \gamma-3)} \\
& \pi_{1}=\frac{(16 t \gamma-1)(6 t \gamma+2 d \gamma-1)^{2}}{4 \gamma(16 t \gamma-3)^{2}}, \quad \pi_{2}=\frac{(8 t \gamma-1)(4 t \gamma-4 d \gamma-1)^{2}}{4 \gamma(16 t \gamma-3)^{2}} .
\end{aligned}
$$

Only firm 2 employs TP: The profits in the second stage are given as

$$
\pi_{1}=\frac{\left(t+d-e_{2}+e_{1}\right)^{2}}{8 t}-\gamma e_{1}^{2}, \quad \pi_{2}=\frac{\left(3 t-d-e_{1}+e_{2}\right)^{2}}{16 t}-\gamma e_{2}^{2} .
$$

The first-order conditions yield the following reaction functions of the firms:

$$
e_{1}\left(e_{2}\right)=\frac{t+d-e_{2}}{8 t \gamma-1}, \quad e_{2}\left(e_{1}\right)=\frac{3 t-d-e_{1}}{16 t \gamma-1}
$$


Table 2: Payoff matrix in the full game

\begin{tabular}{c|c|c}
$1 / 2$ & $\mathrm{UP}$ & $\mathrm{TP}$ \\
\hline $\mathrm{UP}$ & $\frac{(18 t \gamma-1)(9 t \gamma-3 d \gamma-1)^{2}}{36 \gamma(9 t \gamma-1)^{2}}$ & $\frac{(16 t \gamma-1)(6 t \gamma-2 d \gamma-1)^{2}}{4 \gamma(16 t \gamma-3)^{2}}$ \\
\hline & $\frac{(18 t \gamma-1)(9 t \gamma+3 d \gamma-1)^{2}}{36 \gamma(9 t \gamma-1)^{2}}$ & $\frac{(8 t \gamma-1)(4 t \gamma+4 d \gamma-1)^{2}}{4 \gamma(16 t \gamma-3)^{2}}$ \\
\hline $\operatorname{TP}$ & $\frac{(8 t \gamma-1)(4 t \gamma-4 d \gamma-1)^{2}}{4 \gamma(16 t \gamma-3)^{2}}$ & $\frac{(4 t \gamma-1)(2 t \gamma-2 d \gamma-1)^{2}}{16 \gamma(2 t \gamma-1)^{2}}$ \\
\hline & $\frac{(16 t \gamma-1)(6 t \gamma+2 d \gamma-1)^{2}}{4 \gamma(16 t \gamma-3)^{2}}$ & $\frac{(4 t \gamma-1)(2 t \gamma+2 d \gamma-1)^{2}}{16 \gamma(2 t \gamma-1)^{2}}$ \\
\hline
\end{tabular}

Solving these equations, we have

$$
\begin{aligned}
& e_{1}=\frac{4 t \gamma+4 d \gamma-1}{2 \gamma(16 t \gamma-3)}, \quad e_{2}=\frac{6 t \gamma-2 d \gamma-1}{2 \gamma(16 t \gamma-3)} \\
& \pi_{1}=\frac{(8 t \gamma-1)(4 t \gamma+4 d \gamma-1)^{2}}{4 \gamma(16 t \gamma-3)^{2}}, \quad \pi_{2}=\frac{(16 t \gamma-3)(6 t \gamma-2 d \gamma-1)^{2}}{4 \gamma(16 t \gamma-3)^{2}} .
\end{aligned}
$$

No firm employs TP: The profits in the second stage are given as

$$
\pi_{1}=\frac{\left(3 t+d-e_{2}+e_{1}\right)^{2}}{18 t}-\gamma e_{1}^{2}, \quad \pi_{2}=\frac{\left(3 t-d-e_{1}+e_{2}\right)^{2}}{18 t}-\gamma e_{2}^{2} .
$$

The first-order conditions yield the following reaction functions of the firms:

$$
e_{1}\left(e_{2}\right)=\frac{3 t+d-e_{2}}{18 t \gamma-1}, \quad e_{2}\left(e_{1}\right)=\frac{3 t-d-e_{1}}{18 t \gamma-1}
$$

Solving these equations, we have

$$
\begin{aligned}
& e_{1}=\frac{9 t \gamma+3 d \gamma-1}{6 \gamma(9 t \gamma-1)}, \quad e_{2}=\frac{9 t \gamma-3 d \gamma-1}{6 \gamma(9 t \gamma-1)} \\
& \pi_{1}=\frac{(18 t \gamma-1)(9 t \gamma+3 d \gamma-1)^{2}}{36 \gamma(9 t \gamma-1)^{2}}, \quad \pi_{2}=\frac{(18 t \gamma-1)(9 t \gamma-3 d \gamma-1)^{2}}{36 \gamma(9 t \gamma-1)^{2}}
\end{aligned}
$$

Payoff matrix: From the above discussion, we obtain the payoff matrix in Table 2. 


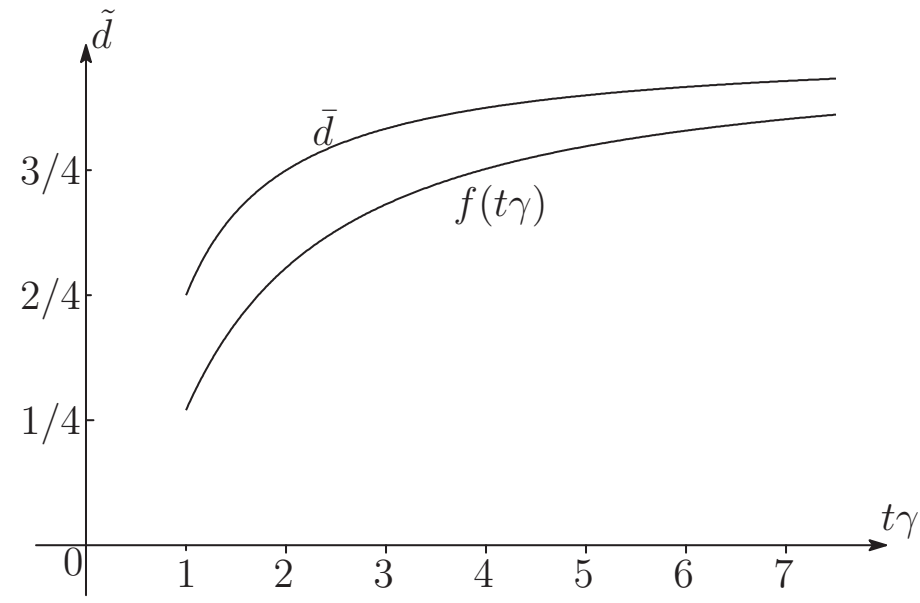

Figure 1: Condition under which firm 2 does not employ TP

We easily find that for firm 1, employing TP dominates employing UP. Given this property, whether firm 2 employs TP depends on the exogenous parameters. We define $\tilde{d} \equiv d / t$. $\tilde{d}$ denotes the cost difference normalized by per length transport cost, $t$. We assume that $\gamma d<t \gamma-1 / 2$ to ensure that the quantities supplied by the firms are always positive. The inequality is rewritten as $\tilde{d}<1-1 /(2 t \gamma) \equiv \bar{d}$. $\bar{d}$ is the upper bound of $\tilde{d}$.

A simple comparison leads to the following proposition.

Proposition 1. (i) If $\tilde{d}>f(t \gamma)$, then only firm 1 employs TP; otherwise, both firms employ TP, where

$f(t \gamma) \equiv \frac{(2 t \gamma-1)\left[(4 t \gamma-1)\left(128 t^{2} \gamma^{2}-16 t \gamma+1\right)-2(16 t \gamma-3) \sqrt{(4 t \gamma-1)(8 t \gamma-1)}\right]}{2 t \gamma\left(512 t^{3} \gamma^{3}-64 t^{2} \gamma^{2}-60 t \gamma+7\right)}$

(ii) The inequality $\bar{d}>f(t \gamma)$ always holds.

Figure 1 indicates the range over which $\bar{d}>\tilde{d}>f(t \gamma)$. Note that as $t \gamma \rightarrow \infty$, both $f(t \gamma)$ and $\bar{d}$ converge to 1 .

When both firms employ TP, a cost reduction implemented by firm $i$ does not change firm $j$ 's prices for the points that firm $i$ supplies. This implies that the cost- 
reducing activities of firm $i$ do not accelerate the price competition between the two firms. That is, the strategic effect of cost-reducing activities is quite different from that in the standard price competition model. Under the strategic environment, the expected market share of a firm is the only factor that affects the incentive of that firm's cost-reducing activities. The more efficient the firm, the greater is the output over which it can apply the outcomes of its cost-reducing activities, and hence, the greater are its returns from such activities. Because the converse also holds, the incentive of the inefficient firm to engage in cost-reducing activities gradually diminishes as the ex ante cost difference increases.

When the efficient firm employs TP and the inefficient firm employs UP, a cost reduction implemented by the efficient firm changes the inefficient firm's price. As in the standard price competition model, the cost reduction of the efficient firm accelerates the price competition between them. This pricing strategy of the inefficient firm diminishes the incentive of the efficient firm to engage in cost-reducing activities. This effect is stronger when the ex ante cost difference is large because the efficient firm obtaining a larger market share has a stronger incentive to engage in cost-reducing activities. This implies that employing UP is beneficial for the inefficient firm when the ex ante cost difference is large. Therefore, when $\tilde{d}$ is large enough, the inefficient firm employs UP. Employing TP is not always a dominant strategy for firms even though it is costless.

We check the market share of firm 1 under TP-TP and TP-UP when $\tilde{d}=f(t \gamma)$ (see Figure 2). This would be helpful to understand the situation in which firm 2 should employ UP. Firm 1's market share where $\tilde{d}=f(t \gamma)$ is smaller when $t \gamma$ is smaller. This reflects the fact that the stronger the incentive of firm 1 to reduce its cost, the stronger is the incentive of firm 2 to mitigate the cost-reducing activity by firm 1. Figure 2 also indicates that the equilibrium market share of firm 2 is smaller if it chooses UP instead of TP given that firm 1 chooses TP. 


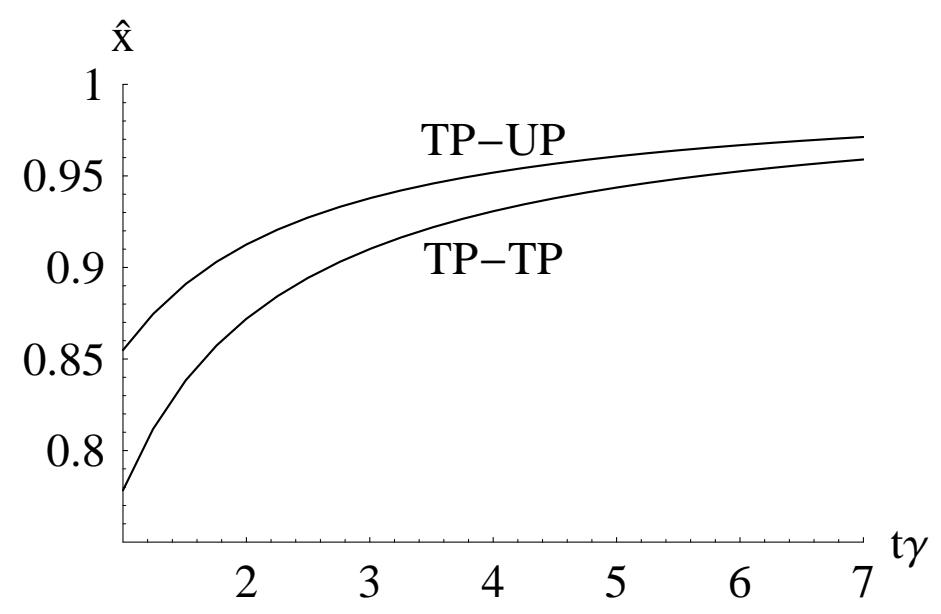

Figure 2: Market share of firm 1 when $\tilde{d}=f(t \gamma)$.

Note: "TP-TP" indicates the case where both firms employ TP, and "TP-UP" indicates the case where only firm 1 employs TP.

We have discussed the case in which firms engage in cost-reducing activities. We can develop a model where they engage in quality-improving activities, which is mathematically equivalent to the model discussed in this subsection. Consider the following model. The marginal cost of the firm is $c$. The product quality of firm 1 is $q_{1}=v+d+e_{1}$ and that of firm 2 is $q_{2}=v+e_{2}$, where $v$ is a positive constant and sufficiently large, $d(>0)$ is the initial quality advantage of firm 1 , and $e_{i}$ is firm $i$ 's effort level to raise the quality of its product. Consumer $x$ buys a good from firm 1 if and only if $q_{1}-p_{1}(x)-t x \geq q_{2}-p_{2}(x)-t(1-x) ;$ note that if $q_{1}-p_{1}(x)-t x=q_{2}-p_{2}(x)-t(1-x)$, the consumer randomly chooses one of the firms. The remaining assumptions are the same to those of the previous model. In this model, we can derive exactly the same payoff matrix in Table 2, and thus, we obtain the following result. ${ }^{8}$

Corollary. Firm 1 (ex ante higher-quality firm) always adopts TP, while firm 2 (ex ante lower-quality firm) adopts $U P$ if $\tilde{d}>f(t \gamma)$.

\footnotetext{
8 The mathematical procedure to derive the result is available upon request.
} 
This is a contrasting result to Choudhary et al. (2005). They examine a vertically differentiated duopoly and show that a higher-quality firm can be worse off with TP, while a lower-quality firm cannot. There are three important differences between our model and theirs. First, in our model horizontal product differentiation exists. Second, we assume that quality improvement requires fixed cost, while they assume that it raises a marginal cost. Third, most importantly, in our model, an increase in the product quality in a firm raises the willingness to pay for consumers uniformly, while in the model of Choudhary et al. (2005), an increase in the product quality in a firm raises the willingness to pay for consumers unequally, more so for consumers who have a higher preference for high quality.

Consider a standard vertical differentiation model discussed by them. Suppose that firm 1 is the higher-quality firm and firm 2 is the lower-quality firm. Firm 1 (firm 2) supplies the product for consumers whose willingness to pay for a quality improvement is relatively high (low). Consider the uniform pricing policy. Suppose that firm 1 improves its quality. This quality improvement increases the willingness to pay for consumers unequally, more so for consumers who have a higher preference for high quality, and less for marginal consumers. Firm 1 raises the price more than the increase in willingness to pay for marginal consumers and reaps more profits from consumers whose loyalty to firm 1 is high. As a result, competition between two firms is mitigated. On the contrary, a quality improvement in firm 2's product increases the willingness to pay more for marginal consumers over whom firms compete than for the consumers whose loyalty to firm 2 is high. As a result, competition is accelerated. Consider the personalized pricing policy. An increase in the quality of each firm's product improves the competitive advantage more for consumers whose loyalty to firm 1 is higher. Thus, the effect of an increase in the quality of firm 1 on firm 2's profit is weak because the effect on firm 2's consumers is weak. On the contrary, the effect of an 
increase in the quality of firm 2 on firm 1's profit is strong because the effect on firm 1's consumers is strong. In other words, the strategic effects of quality improvement are asymmetric in both policies. For these reasons, the profit loss of firm 1 by the rival's quality improvement is significant for firm 1, and thus, firm 1 adopts UP to dampen the quality-improvement activity by firm 2 .

In our model, quality improvements by both the higher-quality and the lower-quality firms accelerate competition, which is unlike in the above vertical differentiation model. In our model, the higher-quality firm is the firm with a larger market share, and the lower-quality firm has a stronger incentive for dampening the quality-improvement activity by the rival whose market share is large, as is discussed in the cost-reducing model. Thus, the lower-quality firm adopts UP for this strategic purpose.

\subsection{Asymmetric effort costs}

We consider the case in which the effort costs to decrease the marginal cost depend on the decision of pricing policy. We only mention the additional assumption on the model in the previous subsection. We assume that $I\left(e_{i}\right)=\alpha \gamma e_{i}^{2}$ if firm $i$ employs TP, where $\alpha>1$. That is, the effort cost under TP is larger than that under UP. Employing TP requires more skills for the firm. This higher requirement level is also applied to the effort cost under TP. We therefore assume that $\alpha>1$. To guarantee that the secondorder conditions for cost-reducing activities are satisfied, we assume that $\alpha t \gamma \geq 1$. The timing structure of the game is the same as in the previous subsection.

The decision of firm 2 to employ TP depends on the ex ante cost difference between the firms, $\tilde{d}$, as depicted in Figure 1. The threshold value of $\tilde{d}$ depends not only on $t \gamma$ but also on the difficulty of cost reduction under TP, $\alpha$. Denote the threshold value by $f(\alpha t \gamma)$. We have the following result.

Proposition 2. (i) Firm 1 always employs TP, and firm 2 does if and only if 

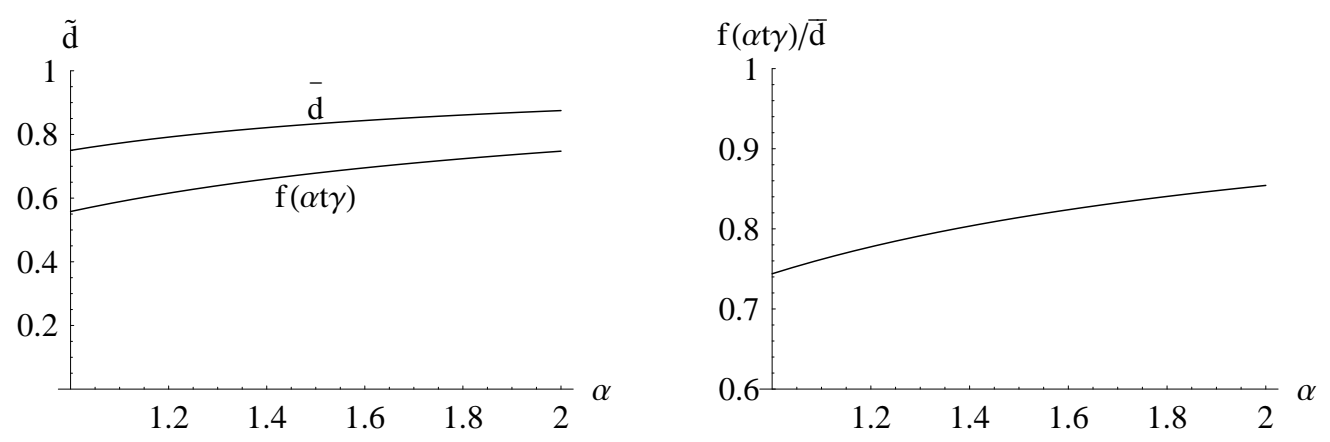

Figure 3: Threshold value of $\tilde{d}, f(\alpha t \gamma)$.

Note: We fix $t \gamma$ at 2 for presentation. $\bar{d}$ is the upper bound of $\tilde{d}$.

$\tilde{d}<f(\alpha t \gamma)$. (ii) $f(\alpha t \gamma)$ is increasing in $\alpha$.

This means that as cost reduction under TP becomes more difficult, firm 2's incentive to employ TP increases (see Figure 3). As explained earlier, the key point of our model is that the employment of TP by firm 2 enhances the incentive of firm 1 for cost-reducing activity given that firm 1 employs TP. An increase in the difficulty of cost reduction (an increase in $\alpha$ ) weakens this strategic reaction of firm 1 to firm 2's adoption of TP, which enhances the incentive of firm 2 to employ TP.

\subsection{Pricing policy and marginal costs}

We have assumed that employing TP does not increase the firm's marginal cost. Instead we assume that the effort costs to decrease the marginal cost depend on the decision of pricing policy, we briefly discuss the case in which the decision of pricing policy increases the ex ante marginal cost. We only mention the additional assumption on the model in the basic model. Assume that the ex ante marginal cost of firm 1 is $c_{1}=c+f-e_{1}$ if it employs TP otherwise this is $c_{1}=c-e_{1}$. Assume also that the ex ante marginal cost of firm 2 is $c_{2}=c+d+f-e_{2}$ if it employs TP otherwise this is $c_{2}=c+d-e_{2}$. Furthermore, for analytical simplicity, we also assume that $f<d$, 
that is, the marginal cost increment through employing TP is not large. The timing structure of the game is the same as in the previous subsection. Under the modified setting, we find that firm 2 does not employ TP for wider parameter ranges because no employment of TP has additional value for firm 2. This functions as a marginal cost reducing investment. By no employment of $\mathrm{TP}$, the ex ante marginal cost of firm 2 is $c_{2}=c+d-e_{2}$ instead of $c_{2}=c+d+f-e_{2}$. The additional benefit induces firm 2 not to employ TP.

\section{Conclusion}

The recent developments in IT enable firms to employ personalized pricing (Arora et al., 2008 and references therein). Following the recent trend, this paper provides a simple model to investigate whether firms should employ personalized pricing. The model setting in this paper is based on those of Thisse and Vives (1988) and Shaffer and Zhang (2002). Our model incorporates the fact that firms engage in cost-reducing activities after they determine their pricing policies. The main result of this paper is that when the ex ante cost difference between the firms is large, employing personalized pricing harms the less-efficient firm even though employing this pricing is costless. This result does not hold when the firms do not engage in cost-reducing activities. That is, when the ex post activities related to cost reductions are important to firms, lessefficient firms do not have to employ personalized pricing. This result has a managerial implication for firms that consider whether to employ personalized pricing. Our main result implies that such firms should take into account their relative competitive positions and technological environments. When firms are small and their cost structures is easily changed by their efforts, they would need to reconsider whether to employ personalized pricing. 


\section{References}

[1] Alptekinoğlu, Aydın and Corbett, Charles J., 2008. Mass customization vs. mass production: Variety and price competition. Manufacturing and Service Operations Management 10(2), 204-217.

[2] Arora, Neeraj; Dreze, Xavier; Ghose, Anindya; Hess, James D; Iyengar, Raghuram; Jing, Bing; Joshi, Yogesh; Kumar, V.; Lurie, Nicholas; Neslin, Scott; Sajeesh, S.; Su, Meng; Syam, Niladri; Thomas, Jacquelyn and Zhang, Z. John, 2008. Putting one-to-one marketing to work: Personalization, customization and choice. Marketing Letters 19(3-4), 305-321.

[3] Banks, Jeffrey and Sridhar Moorthy, 1999. A model of price promotions with consumer search. International Journal of Industrial Organization 17(3), 371-398.

[4] Bester, Helmut and Petrakis, Emmanuel, 1996. Coupons and oligopolistic price discrimination. International Journal of Industrial Organization 14(2), 227-242.

[5] Bhaskar, V. and To, Ted, 2004. Is perfect price discrimination really efficient? an analysis of free entry, RAND Journal of Economics 35(4), 762-776.

[6] Brander, James A. and Spencer, Barbara J., 1983. Strategic commitment with R\&D: The symmetric case. Bell Journal of Economics 14(1), 225-235.

[7] Chen, Yuxin and Iyer, Ganesh, 2002. Consumer addressability and customized pricing. Marketing Science 21(2), 197-208.

[8] Choudhary, Vidyanand; Ghose, Anindya; Mukhopadhyay, Tridas and Rajan, Uday, 2005. Personalized pricing and quality differentiation. Management Science 51(7), 1120-1130.

[9] Cohen, Wesley M. and Klepper, Steven, 1996. A Reprise of Size and R\&D. Economic Journal 106(437), 925-951.

[10] Desai, Preyas S. and Purohit, Devavrat, 2004. "Let me talk to my manager": Haggling in a competitive environment. Marketing Science 23(2), 219-233. 
[11] Dogan, Kutsal; Haruvy, Ernan and Rao, Ram C., 2010. Who should practice price discrimination using rebates in an asymmetric duopoly? Quantitative Marketing and Economics 8(1), 61-90.

[12] Fudenberg, Drew and Tirole, Jean, 2000. Customer poaching and brand switching. RAND Journal of Economics 31(4), 634-657.

[13] Ghose, Anindya and Huang, Ke-Wei, 2009. Personalized pricing and quality customization. Journal of Economics and Management Strategy 18(4), 1095-1135.

[14] Ishida, Junichiro; Matsumura, Toshihiro; Matsushima, Noriaki, 2011. Market competition, R\&D and firm profits in asymmetric oligopoly. Journal of Industrial Economics 59(3), 484-505.

[15] Liu, Qihong and Serfes, Konstantinos, 2004. Quality of information and oligopolistic price discrimination. Journal of Economics and Management Strategy 13(4), $671-702$.

[16] Liu, Yunchuan and Zhang, Z. John, 2006. The benefits of personalized pricing in a channel. Marketing Science 25(1), 97-105.

[17] Matsumura, Toshihiro and Matsushima, Noriaki, 2010. When small firms fight back against large firms in R\&D activities. The B.E. Journal of Economic Analysis \& Policy 10(1) (Topics), Article 81.

[18] Moorthy, Sridhar, 2005. A general theory of pass-through in channels with category management and retail competition. Marketing Science 24(1), 110-122.

[19] Rao, Ram C., 1991. Pricing and promotions in asymmetric duopolies. Marketing Science, 10(2), 131-144.

[20] Raju, Jagmohan and Zhang, Z. John, 2010. Smart Pricing: How Google, Priceline, and Leading Businesses Use Pricing Innovation for Profitability. Pearson Prentice Hall, Upper Saddle River, NJ. 
[21] Shaffer, Greg and Zhang, Z. John, 1995. Competitive coupon targeting. Marketing Science 14(4), 395-416.

[22] Shaffer, Greg and Zhang, Z. John, 2000. Pay to switch or pay to stay: Preferencebased price discrimination in markets with switching costs. Journal of Economics and Management Strategy 9(3), 397-424.

[23] Shaffer, Greg and Zhang, Z. John, 2002. Competitive one-to-one promotions. Management Science 48(9), 1143-1160.

[24] Shulman, Jeffrey D. and Geng, Xianjun, 2012. Add-on pricing by asymmetric firms. forthcoming in Management Science.

http://dx.doi.org/10.1287/mnsc.1120.1603

[25] Taylor, Curtis R., 2004, Consumer privacy and the market for customer information. RAND Journal of Economics 35(4), 631-650.

[26] Thisse, Jacques-François and Vives, Xavier, 1988. On the strategic choice of spatial price policy. American Economic Review 78(1), 122-137.

[27] Villas-Boas, J. Miguel, 1999, Dynamic competition with customer recognition. RAND Journal of Economics 30(4), 604-631.

[28] Zhang, Z. John, 2009. Competitive targeted pricing: perspectives from theoretical research. Vithala R. Rao, ed. Handbook of Pricing Research in Marketing. Edward Elgar Publishing, Northampton, MA, 302-318. 


\section{Appendix (not for publication)}

We explain the calculation to derive Corollary in Section 4.1. There are four cases depending on the pricing strategies set by the firms. We discuss each of the four cases.

UP-UP When both firms employ UP, the indifferent consumer, $\hat{x}_{n n}$, is given as

$$
q_{1}-p_{1}-t \hat{x}_{n n}=q_{2}-p_{2}-t\left(1-\hat{x}_{n n}\right) \quad \rightarrow \quad \hat{x}_{n n}=\frac{t+q_{1}-q_{2}+p_{2}-p_{1}}{2 t}
$$

The profits are given by

$$
\pi_{1}=\left(p_{1}-c\right) \hat{x}_{n n}, \quad \pi_{2}=\left(p_{2}-c\right)\left(1-\hat{x}_{n n}\right)
$$

The first-order conditions lead to

$$
p_{i}=\frac{3 t+3 c+q_{i}-q_{j}}{3}, \quad \hat{x}_{n n}=\frac{3 t+q_{1}-q_{2}}{6 t}, \quad \pi_{i}=\frac{\left(3 t+q_{i}-q_{j}\right)^{2}}{18 t}
$$

By substituting $q_{1}=v+d+e_{1}$ and $q_{2}=v+e_{2}$ into $\pi_{i}$, we have

$$
\pi_{1}=\frac{\left(3 t+d+e_{1}-e_{2}\right)^{2}}{18 t}, \quad \pi_{2}=\frac{\left(3 t-d-e_{1}+e_{2}\right)^{2}}{18 t} .
$$

The profits are equivalent with those under 'No firm employs TP' in Section 4.1 except the effort costs, $\gamma e_{i}^{2}$.

UP-TP Consider the case in which firm 1 employs UP and firm 2 employs TP. We can apply the discussion of this case to that in which firm 2 employs UP and firm 1 employs TP. When only firm 1 employs UP, the timing is as follows. First, firm 1 sets its price. Second, observing this price, firm 2 sets its prices.

Given that firm 1 sets its price $p_{1}$, firm 2 takes the demand of consumer $x$ if and only if

$$
q_{1}-p_{1}-t x<q_{2}-p_{2}(x)-t(1-x) \quad \rightarrow \quad p_{2}(x)<q_{2}-q_{1}+p_{1}+t(2 x-1) .
$$


If the right-hand side in the latter inequality is larger than the marginal cost of firm 2 , $c_{2}$, it sets $p_{2}(x)$ at $q_{2}-q_{1}+p_{1}+t(2 x-1)-\varepsilon$ to take the demand of consumer $x$, where $\varepsilon$ is sufficiently small; we omit this value. Otherwise, it sets $p_{2}(x)$ at $c_{2}$. The optimal price of firm 2 at point $x$ is given by

$$
p_{2}(x)= \begin{cases}q_{2}-q_{1}+p_{1}+t(2 x-1) & \text { if } c<q_{2}-q_{1}+p_{1}+t(2 x-1), \\ c & \text { otherwise. }\end{cases}
$$

The indifferent consumer $\hat{x}_{n d}$ is given by

$$
c=q_{2}-q_{1}+p_{1}+t(2 x-1) \quad \rightarrow \quad \hat{x}_{n d}=\frac{t+c+q_{1}-q_{2}-p_{1}}{2 t} .
$$

Anticipating the prices of firm 2, firm 1 sets its price, $p_{1}$. The profit of firm 1 is given by

$$
\pi_{1}=\left(p_{1}-c\right) \hat{x}_{n d}=\frac{\left(p_{1}-c\right)\left(t+c+q_{1}-q_{2}-p_{1}\right)}{2 t} .
$$

The first-order condition leads to

$$
p_{1}=\frac{t+2 c+q_{1}-q_{2}}{2}, \quad \hat{x}_{n d}=\frac{t+q_{1}-q_{2}}{4 t}, \quad \pi_{1}=\frac{\left(t+q_{1}-q_{2}\right)^{2}}{8 t} .
$$

The profit of firm 2 is given by

$$
\pi_{2}=\int_{\hat{x}_{n d}}^{1}\left(q_{2}-q_{1}+p_{1}+t(2 m-1)-c\right) d m=\frac{\left(3 t+q_{2}-q_{1}\right)^{2}}{16 t} .
$$

By substituting $q_{1}=v+d+e_{1}$ and $q_{2}=v+e_{2}$ into $\pi_{i}$, we have

$$
\pi_{1}=\frac{\left(t+d+e_{1}-e_{2}\right)^{2}}{8 t}, \quad \pi_{2}=\frac{\left(3 t+e_{2}-d-e_{1}\right)^{2}}{16 t} .
$$

The profits are equivalent with those under 'Only firm 2 employs TP' in Section 4.1 except the effort costs, $\gamma e_{i}^{2}$.

Conversely, under the case in which firm 2 employs UP and firm 1 employs TP, the profits of the firms are given by

$$
\pi_{1}=\frac{\left(3 t+q_{1}-q_{2}\right)^{2}}{16 t}, \quad \pi_{2}=\frac{\left(t+q_{2}-q_{1}\right)^{2}}{8 t} .
$$


By substituting $q_{1}=v+d+e_{1}$ and $q_{2}=v+e_{2}$ into $\pi_{i}$, we have

$$
\pi_{1}=\frac{\left(3 t+d+e_{1}-e_{2}\right)^{2}}{16 t}, \quad \pi_{2}=\frac{\left(t+e_{2}-d-e_{1}\right)^{2}}{8 t} .
$$

The profits are equivalent with those under 'Only firm 1 employs TP' in Section 4.1 except the effort costs, $\gamma e_{i}^{2}$.

TP-TP When both firms employ TP, the lowest price of firm $i$ for each consumer is $c$. Firm 1 takes the demand of consumer $x$ if and only if

$$
q_{1}-p_{1}(x)-t x>q_{2}-c-t(1-x) \rightarrow p_{1}(x)<q_{1}-q_{2}+c+t(1-2 x) .
$$

If the right-hand side in the latter inequality is larger than the marginal cost of firm 1 , $c$, it sets $p_{1}(x)$ at $q_{1}-q_{2}+c+t(1-2 x)-\varepsilon$ to take the demand of consumer $x$, where $\varepsilon$ is sufficiently small; we omit this value. Otherwise, it sets $p_{1}(x)$ at $c$. The optimal price of firm 1 at point $x$ is given by

$$
p_{1}(x)= \begin{cases}q_{1}-q_{2}+c+t(1-2 x) & \text { if } q_{2}<q_{1}+t(1-2 x) \\ c & \text { otherwise }\end{cases}
$$

A similar argument is applied to the prices of firm 2 .

$$
p_{2}(x)= \begin{cases}q_{2}-q_{1}+c+t(2 x-1) & \text { if } q_{1}<q_{2}+t(2 x-1), \\ c & \text { otherwise }\end{cases}
$$

The indifferent consumer $\hat{x}_{d d}$ is given by

$$
q_{1}-c-t x=q_{2}-c-t(1-x) \rightarrow \hat{x}_{d d}=\frac{t+q_{1}-q_{2}}{2 t} .
$$

The profits of the firms are given by

$$
\begin{aligned}
& \pi_{1}=\int_{0}^{\hat{x}_{d d}}\left(q_{1}-q_{2}+c+t(1-2 m)-c\right) d m=\frac{\left(t+q_{1}-q_{2}\right)^{2}}{4 t}, \\
& \pi_{2}=\int_{\hat{x}_{d d}}^{1}\left(q_{2}-q_{1}+c+t(2 m-1)-c\right) d m=\frac{\left(t+q_{2}-q_{1}\right)^{2}}{4 t} .
\end{aligned}
$$


By substituting $q_{1}=v+d+e_{1}$ and $q_{2}=v+e_{2}$ into $\pi_{i}$, we have

$$
\pi_{1}=\frac{\left(t+d+e_{1}-e_{2}\right)^{2}}{4 t}, \quad \pi_{2}=\frac{\left(t+e_{2}-d-e_{1}\right)^{2}}{4 t} .
$$

The profits are equivalent with those under 'Both firms employ TP' in Section 4.1 except the effort costs, $\gamma e_{i}^{2}$. 